\title{
The Spin of the Proton
}

\author{
A. W. Thomas ${ }^{1,2}$ \\ ${ }^{1}$ Jefferson Lab, 12000 Jefferson Ave., Newport News VA 23606 USA \\ ${ }^{2}$ College of William and Mary, Williamsburg VA 23187 USA
}

October 29, 2018

\begin{abstract}
The twenty years since the announcement of the proton spin crisis by the European Muon Collaboration has seen tremendous progress in our knowledge of the distribution of spin within the proton. The problem is reviewed, beginning with the original data and the suggestion that polarized gluons may play a crucial role in resolving the problem through the U(1) axial anomaly. The discussion continues to the present day where not only have strong limits have been placed on the amount of polarized glue in the proton but the experimental determination of the spin content has become much more precise. It is now clear that the origin of the discrepancy between experiment and the naive expectation of the fraction of spin carried by the quarks and anti-quarks in the proton lies in the non-perturabtive structure of the proton. We explain how the features expected in a modern, relativistic and chirally symmetric description of nucleon structure naturally explain the current data.
\end{abstract}

\section{Introduction}

In our quest to understand the structure of the nucleon and eventually of nuclei at the fundamental level of quarks and gluons, few problems have proven more exciting than that of mapping the distribution of the spin of the proton onto these constituents [1, 2]. This began in earnest with the announcement by the European Muon Collaboration (EMC) [3] in 1988 that the quarks carried only a very small fraction of the proton spin, possibly zero. This soon became known as the "proton spin crisis".

Several effects which were based on well known features of hadron structure were explored [4, 5] but none were able to produce such a small number. On the other hand, it was soon realized that, because of the famous $U(1)$ axial anomaly [6, 7], polarized gluons could also make a contribution to the proton spin structure function [8, 9, 10. Indeed, it was shown that if the gluons in a polarized proton were to carry 4 units of spin, the EMC spin crisis would be resolved. No believable explanation of how the gluons might carry 4 units of spin was ever given. Nevertheless, the mathematical beauty of the proposed resolution was seductive and major experimental programs were launched to investigate it.

Over the past twenty years that experimental effort has produced spectacular results, with remarkable increases in precision and new kinds of data which probe for the presence of polarized gluons directly. There have also been some remarkable advances in our ability to solve QCD on a lattice of points in space-time and extract new information about nucleon structure. Indeed, the progress made has reached the stage where it is possible to assert that the gross features of the proton spin problem are now understood. This does not mean that considerable experimental and theoretical effort will not be required to confirm the resolution. However, as we shall explain, well known and understood aspects 
of hadron structure, in combination with the totality of modern experimental evidence leave little room for doubt.

The structure of this lecture is that we first review the experimental situation for the proton spin structure function as well as the search for polarized glue - all in the context of the explanation proposed in terms of the axial anomaly. We then review the possible explanations in terms of more conventional aspects of hadron structure which were suggested within a few months of the EMC preprint. These explanations are then examined in the light of modern insights into hadron structure coming from the study of their properties as a function of quark mass using lattice QCD. We then close by summarizing the resolution of the spin problem and the future experimental work needed to confirm it.

\section{The Experimental Data}

The major advance made by the EMC was to extend the measurements of the proton spin structure function, $g_{1}^{p}\left(x, Q^{2}\right)$, to a value of Bjorken- $x$ almost as low as $10^{-2}$. This enabled a more accurate evaluation of the integral of the spin structure function. The latter can be rigorously expressed in terms of well known, perturbative QCD corrections and three low energy constants, the axial charges $g_{A}^{(3)}$ and $g_{A}^{(8)}$, which are known from neutron and hyperon $\beta$-decay, as well as the quantity $\Sigma$ (often written as $\Delta \Sigma$ or $\left.a_{0}\right) 1$ Within the parton model $\Sigma=\Delta u+\Delta d+\Delta s$, with $\Delta q=\int_{0}^{1} \Delta q(x) d x$, the fraction of the helicity of the proton carried by the quarks and anti-quarks of flavor $q$. (We stress that while the word spin can be ambiguous, in the context of the spin problem it rigorously refers to the helicity of quarks in a proton, in the infinite momentum frame, with positive helicity.)

For clarity we simply denote the perturbative QCD coefficients, which are power series expansions in the strong coupling constant, $\alpha_{s}\left(Q^{2}\right)$, as $c_{i}\left(Q^{2}\right)$. Then the sum rule for the proton spin structure function is:

$$
\int_{0}^{1} d x g_{1}^{p}\left(x, Q^{2}\right)=\frac{1}{12} c_{1}\left(Q^{2}\right) g_{A}^{(3)}+\frac{1}{36} c_{1}\left(Q^{2}\right) g_{A}^{(8)}+\frac{1}{9} c_{2}\left(Q^{2}\right) \Sigma .
$$

Since $g_{A}^{(3,8)}$ are well known, having an accurate experimental determination of the left-hand side allows one to deduce $\Sigma$. The result of the EMC measurement was

$$
\Sigma=14 \pm 3 \pm 10 \%
$$

which is clearly incompatible with $100 \%$, or even $65 \%$, which was the more realistic target after accounting for the relativistic motion of the quarks expected, for example, from bag model studies [1] see also Ref. [12] for a similar result in a modern, relativistic confining model. Psychologically, it was even more important that this result was consistent with zero and this led to considerable excitement concerning the Skyrme model, which was initially believed to make just such a prediction. We note that it is now known that this is not the case and furthermore, as we shall see, the experimental data is no longer compatible with zero.

In modern terms Eq. (1) is especially interesting as one can rigorously extract $\Sigma$ from a measurement of the axial weak, neutral current charge of the proton after correcting for heavy quark radiative effects [13]. In this way it becomes a very interesting sum rule which can really only be violated if there is $\delta$-function contribution at $x=0$ - as proposed by Bass [14] in connection with the fascinating idea that some of the spin of a constituent quark might be associated with topological charge stored in its gluon fields. At the time, the value of $\Sigma$ was immediately seen to violate the Ellis-Jaffe sum rule, which was based on the idea that if the proton contains no strange quarks one expects $\Sigma=g_{A}^{(8)}$; which is clearly violated by the EMC data. This led to the idea that the proton might have a significant polarized strange sea and that in turn stimulated a number of important experimental programs.

\footnotetext{
${ }^{1}$ Here we denote by $\Sigma$ the renormalization group invariant spin content, $\Sigma\left(\mu^{2}=\infty\right)$. We refer to the discussion in Ref. [30] of the connection between $\Sigma$ calculated in a quark model and $\Sigma\left(\mu^{2}=\infty\right)$.
} 


\subsection{The Possible Role of the U(1) Axial Anomaly}

Although the gluon carries no electric charge, it can create a quark-anti-quark pair which then interact with an incoming photon. If we view the deep-inelastic scattering cross section as the imaginary part of the forward photon-nucleon Compton amplitude, then this gluonic term corresponds to the imaginary part of a quark-anti-quark box diagram. The moments of the structure functions correspond to integrating over the momentum of the quark (or anti-quark) which is struck by the incoming photon and this effectively shrinks the box diagram to a triangle. In the case of spin dependent deep-inelastic scattering, the corresponding integral is quadratically divergent and the answer depends on whether or not the regularization procedure respects gauge invariance or chiral symmetry; it cannot respect both.

Thus, if one works within a scheme that ensures chiral symmetry, the renormalized quark spin operators satisfy the usual $\mathrm{SU}(2)$ commutation relations $\left(\left[S_{i}, S_{j}\right]=\epsilon_{i j k} S_{k}\right)$ and the axial current is conserved. On the other hand, if one chooses to ensure the more fundamental symmetry, namely gauge symmetry, the renormalized spin operators do not satisfy the usual commutation relations and the divergence of the renormalized axial current is not zero [14, 15]. In that case, the value of $\Sigma$, defined through the matrix element of the axial charge, renormalized in a gauge invariant manner, can be written:

$$
\Sigma=\Sigma_{\text {naive }}-\frac{N_{f} \alpha_{s}\left(Q^{2}\right)}{2 \pi} \Delta G\left(Q^{2}\right)
$$

Here $\Sigma_{\text {naive }}$ is the spin carried by the quarks in a naive quark model (i.e. one that respects chiral symmetry and ignores the effect of the axial anomaly) and the second term is the contribution arising, through the axial anomaly, from polarized gluons in the proton.

On the face of it, as $\alpha_{s}\left(Q^{2}\right)$ vanishes as $Q^{2}$ approaches $\infty$, it appears naively that the correction term on the right of Eq.(3) should vanish in the Bjorken limit. However, again because of the axial anomaly, the evolution of $\Delta G\left(Q^{2}\right)$ is such that the product, $\alpha_{s}\left(Q^{2}\right) \Delta G\left(Q^{2}\right)$, should go to a non-zero constant as $Q^{2} \rightarrow \infty$. Thus the gluon box diagram must be included in the Bjorken limit.

At a typical scale of $Q^{2}=3 \mathrm{GeV}^{2}$, the strong coupling, $\alpha_{s}\left(Q^{2}\right)$, is around 0.3 . Thus for 3 active flavors of quark the correction to the naive spin content is roughly $0.15 \Delta G$ and if $\Delta G$ were of order 4 , the naive expectation for the fraction of the spin of the proton carried by its quarks, namely about $2 / 3$ after allowance for the relativistic motion of the valence quarks, would be brought into agreement with the EMC data.

It is important to realize a couple of things. First, no-one ever proposed a believable model of nucleon structure in which one could see how the gluons might carry so much spin. Secondly, it was understood that if the gluons carried 4 units of spin they must also carry about 4 units of orbital angular momentum so that the total spin of the nucleon remains at $1 / 2$. This second caution has tended to be forgotten as the limits on the amount of spin carried by gluons have tended to decrease.

Finally, we note that in the case of an explicitly gauge invariant scheme, such as $\overline{\mathrm{MS}}$, there is no explicit gluon correction to $\Sigma$. Instead, the individual values of $\Delta q$ incorporate the effect of the axial anomaly. Whether one one works in a scheme that preserves chiral symmetry or gauge symmetry,

provided the effect of the anomaly is treated consistently, the same physics is incorporated. It just appears in different places.

\subsection{The Shape of the Gluon Contribution to $g_{1}^{p}$}

Although most of the focus on the gluon contribution to the spin structure function of the proton focussed on its first moment, it is also of considerable importance to know the characteristic shape of the contribution from the photon-gluon box diagram to the proton spin structure function [16]. In fact, as emphasized very early by Bass and co-workers, the shape of this contribution is distinctive, with $\delta g_{1}^{p}(x)$ going rapidly large and negative for $x$ below $10^{-2}[15$ - if $\Delta G$ is positive, as originally required to explain the spin crisis. 
We also note that $\Sigma$ is a flavor singlet quantity, so it will be the same for the proton and the neutron. Thus, while the large iso-vector contribution (corresponding to $g_{A}^{(3)}$ in Eq.(11)) cancels in the measurement of the deuteron spin structure function, the contribution of the photon-gluon box diagram is enhanced. This makes the recent, very precise measurements of the deuteron spin structure function down to below $10^{-4}$, by the COMPASS Collaboration [17, 18], especially significant. Those measurements are completely consistent with zero for $x$ from $5 \times 10^{-5}$ to almost $10^{-1}$. This alone puts a very severe constraint on the experimental value of $\Delta G$ - making it impossible for $\Delta G$ to be anywhere near as large as 4 .

At Jefferson Lab, the CLAS Collaboration has more than doubled the number of polarized spin structure function data in the deep-inelastic region. Because of the relatively low range of $Q^{2}$ it is crucial to allow for higher-twist effects in amalyzing the data. Nevertheless, Leader et al. [19] have found that including the JLab data [20] in their global analysis reduces the error on the determination of $x \Delta G$ by a factor of 3 from what is possible without it. Their conclusion is that $|\Delta G|<0.3$.

\subsection{Direct Searches for $\Delta G$}

Another characteristic of the gluon box diagram is that it tends to be dominated by quark-anti-quark pairs with transverse momentum equal to or larger than $Q$ [10]. Thus, one can search directly for the existence of polarized gluons by making semi-inclusive measurements that explicitly look for high- $p_{T}$ hadrons in a deep-inelastic event.

Such direct measurements have been extensively explored at Hermes [21] and COMPASS [22, 23], with negative results. For two different methods of analysis, Hermes has found $\Delta G / G$ to be of order $0.071 \pm 0.034 \pm 0.011$ and $0.071 \pm 0.034 \pm 0.010$ in the low- $x$ region. This led them to the conclusion that $\Delta G$ is small and "unlikely to solve the puzzle of the missing nucleon spin". The COMPASS result is completely consistent with Hermes but even more precise, with the result $\Delta G / G=0.06 \pm 0.31 \pm 0.06$ at $x=0.13$ and $\mu^{2}=3 \mathrm{GeV}^{2}$, using data with $Q^{2}>1 \mathrm{GeV}^{2}$ and looking at high- $p_{T}$ hadrons.

At RHIC both PHENIX [24, 25] and STAR [26, 27] have measured asymmetries in polarized protonproton collisions, either looking for asymmetries (i.e. differences in the cross sections for helicities antiparallel and parallel) in either jet or $\pi^{0}$ production. The preliminary analysis of the latest PHENIX data prefers a value of $\Delta G$ between -0.5 and zero. For STAR the preliminary analysis of Run 6 yielded a limit for $|\Delta G|$ below 0.3 (at $90 \%$ confidence level) and again consistent with zero.

\subsection{Progress in Determining the Integral of $g_{1}^{p}\left(x, Q^{2}\right)$}

The tremendous progress in the measurement of both the proton and neutron spin structure functions over the twenty years since the original EMC discovery have led to a major increase in the accuracy with which the integral of $g_{1}^{p}$ can be determined. We have already mentioned that the range of $x$ over which data exists now extends below $10^{-4}$. In addition, in comparison with the Spin Muon Collaboration, the successor to EMC at CERN which was designed to follow up the discovery of the spin crisis, the latest data is as much as an order of magnitude more precise. The latest analyses of Hermes [28] and COMPASS [29] yield values:

$$
\begin{aligned}
& \Sigma=0.330 \pm 0.011(\text { thry }) \pm 0.025(\exp ) \pm 0.028(\text { evol }) \text { Hermes } \\
& \Sigma=0.33 \pm 0.03(\text { stat }) \pm 0.05(\text { syst }) \quad \text { COMPASS }
\end{aligned}
$$

This represents a very substantial increase in the fraction of the spin of the proton carried by its quarks and anti-quarks. 


\subsection{Summary of the Experimental Situation}

The result of the last twenty years of intense experimental effort has been a very significant improvement in the accuracy with which $\Sigma$ is known. Contrary to the original EMC measurement it is no longer possible for the quarks to carry none of the spin of the proton. Rather it seems that the quarks and anti-quarks in the proton carry about a third of its spin and possibly as much as $40 \%$.

As well as this shift in the target which theory needs to explain, the experimental effort has also established very strong upper limits on the spin of the gluons. It seems that $\Delta G$ cannot be larger than 0.3 and may even be slightly negative. This value of $\Delta G$ is more than an order of magnitude larger than the value (namely 4) originally found necessary to reduce the naive theoretical expectation of $65 \%$ (after allowing for relativistic motion of the valence quarks) to the value observed by EMC, through the axial anomaly. Through Eq. (3) we see that $|\Delta G|<0.3$ implies that through the axial anomaly the gluons yield a correction of less than $5 \%$ to the quark spin content of the proton. Thus the gluon spin is also a factor of 6 too small to explain the difference between $65 \%$ and the current experimental values of $\Sigma$, given in Eq. (44). Indeed, if the hints from PHENIX are right and $\Delta G$ is negative, it may actually make the problem marginally worse, rather than helping to resolve it.

The nature of the challenge presented by the proton spin structure function has changed but it is still a fascinating problem.

\section{The Modern Theoretical Explanation}

The discussion in this section has its origins in two papers written in 1988. The first, written with Andreas Schreiber [4], dealt with the effect of the pion cloud of the nucleon, which is well known to be associated with chiral symmetry. The second, written with Fred Myhrer [5], dealt with what in nuclear physics would be described as an exchange current correction - in this case arising through the hyperfine one-gluon-exchange (OGE) interaction which is usually considered primarily responsible for the difference in mass between the nucleon and $\Delta$, the $\Sigma$ and $\Lambda$ and so on. We consider these two terms in order, noting that the discussion in this section follows closely that presented recently by Myhrer and Thomas [30].

\subsection{The Role of the Pion Cloud}

That virtual pion emission and absorption can play a major role in the properties of hadrons has been appreciated since its existence was proposed by Yukawa. Within the modern context of QCD as the fundamental theory of the strong interaction this remains true, even though we know that the pion is itself composed of quark-anti-quark pairs. Indeed, we understand that the unusually small mass of the pion is associated with the very low values of the $u$ and $d$ quark masses, with $m_{\pi}^{2}$ vanishing linearly as the light quark masses go to zero. That is, it is a pseudo-Goldstone boson associated with the approximate chiral invariance of QCD.

This special property of the pion leads to many important consequences. For example, in the chiral limit (vanishing light quark masses) the charge radius of the proton and neutron go to infinity (plus and minus, respectively). Chiral perturbation theory provides a systematic way of exploring the consequences of chiral symmetry. In particular, it has established that hadron properties, such as charge radii, magnetic moments and so on, are non-analytic functions of the light quark masses solely because of the contributions of Goldstone boson loops. Tracing the non-analytic behaviour of hadron properties has proven a powerful tool in guiding the development of models and in testing whether apparent relationships between physical observables are purely accidental or whether they may be somewhat deeper [31]. 
An unfortunate consequence of the mathematical elegance of the formalism of chiral perturbation theory is that it has been allowed to obscure a number of instances where there is a simple, physically meaningful explanation of some interesting physical phenomena. We recently presented arguments as to why it is both meaningful and even satisfying to think of hadron structure in terms of a pion cloud and we refer interested readers to that discussion [32].

In fact, describing a physical nucleon as having a pion cloud which interacts with the valence quarks of the quark core (the "bare" nucleon), in a manner dictated by the requirements of chiral symmetry, has been very successful in describing the properties of the nucleon [33, 34]. The cloudy bag model (CBM) [33, 34 reflects this description of the nucleon and in this model the nucleon consists of a bare nucleon, $\mid N>$, with a probability $Z \sim 1-P_{N \pi}-P_{\Delta \pi} \sim 0.7$, in addition to being described as a nucleon $(N)$ and a pion and a $\Delta$ and a pion, with probabilities $P_{N \pi} \sim 0.20-0.25$ and $P_{\Delta \pi} \sim 0.05-0.10$, respectively. The phenomenological constraints on these probabilities were discussed, for example, in Refs. [32, 35, 36]. One of the most famous of these constraints is associated with the excess of $\bar{d}$ over $\bar{u}$ quarks in the proton, predicted on the basis of the CBM [37]. Indeed, to first order the integral of $\bar{d}(x)-\bar{u}(x)$ is $2 / 3 P_{N \pi}-P_{\Delta \pi} / 3$, which is consistent with the experimental data [38] if $P_{N \pi}$ and $P_{\Delta \pi}$ lie within the ranges just quoted.

The effect of the pion cloud on the spin carried by quarks was investigated very early by Schreiber and Thomas, who wrote the corrections to the spin sum-rules for the proton and neutron explicitly in terms of the probabilities set out above [4]. For the present purpose it is helpful to rewrite the results of Ref. [4] for the proton and neutron. In fact, if we consider explicitly the flavor singlet combination, proton plus neutron, the pion cloud modifies the quark contribution to the proton spin in the following manner:

$$
\Sigma \rightarrow\left(Z-\frac{1}{3} P_{N \pi}+\frac{5}{3} P_{\Delta \pi}\right) \Sigma
$$

The critical feature of the role of the pion cloud shown in Eq. (5) is that the Clebsch-Gordon algebra for coupling the spin of the nucleon and the orbital angular momentum of the pion in the $N \pi$ Fock state favors a spin down nucleon and a pion with +1 unit of orbital angular momentum in the $z$-direction. This has the effect of replacing quark spin by quark and anti-quark orbital angular momentum. Note that in the $\Delta \pi$ Fock component the spin of the baryon tends to point up (and the pion angular momentum down), thus enhancing the quark spin. Nevertheless, the wave function renormalization factor, $Z$, dominates, yielding a reduction by a factor between 0.7 and 0.8 for the range of probabilities quoted above.

\subsection{The Role of the One-Gluon-Exchange Hyperfine Interaction}

It is well established that the spin-spin interaction between quarks in a baryon, arising from the exchange of a single gluon, explains a major part of the mass difference between the octet and decuplet baryons - e.g., the N- $\Delta$ mass difference [11, 39. This spin-spin interaction must therefore also play a role when an external probe interacts with the three-quark baryon state. That is, the probe not only senses a single quark current but a two-quark current as well. The latter has an intermediate quark propagator connecting the probe and the spin-spin interaction vertices, and is similar to the exchange-current corrections which are well known in nuclear physics.

In the case of spin dependent properties, the probe couples to the various currents in the nucleon. In the first exploration of the two-quark current, illustrated in Fig. 1, carried out by Hogaasen and Myhrer [40], the MIT bag model was used and the quark propagator was written as a sum over quark eigenmodes. The dominant contributions were found to come from the intermediate p-wave anti-quark states.

The primary focus of Ref. [40] was actually the OGE corrections to the magnetic moments and semi-leptonic decays of the baryon octet. For example, this exchange current correction is vital to 


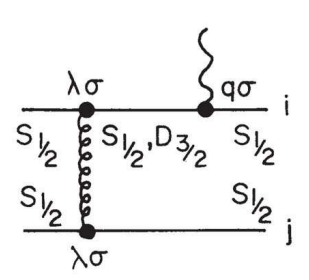

(a)

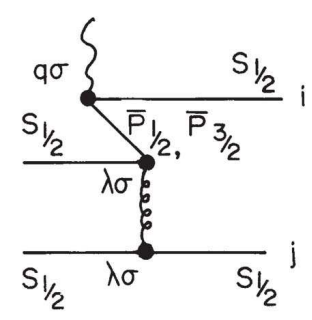

(c)

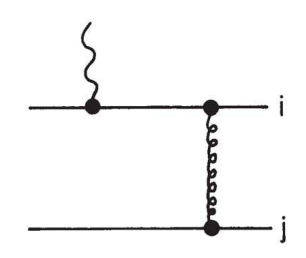

(b)

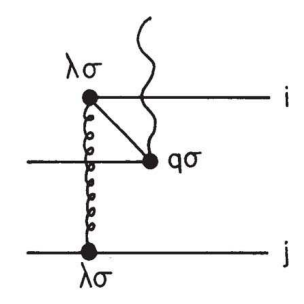

(d)

Figure 1: The one-gluon-exchange correction to the spin sum rule investigated by Myhrer and Thomas [5, 30].

understand the unusual strength of the decay $\Sigma^{-} \rightarrow n+e^{-}+\bar{\nu}_{e}$. Myhrer and Thomas [5] realized the importance of this correction to the flavor singlet axial charge and hence to the proton spin, finding that it reduced the fraction of the spin of the nucleon carried by quarks, calculated in the naive bag model, by 0.15 - i.e., $\Sigma \rightarrow \Sigma-3 G[5]$. The correction term, $G$, is proportional to $\alpha_{s}$ times certain bag model matrix elements [40, where $\alpha_{s}$ is determined by the "bare" nucleon- $\Delta$ mass difference.

As in the case of the correction for relativistic motion of the valence quarks and for the pion cloud, here too the spin lost by the quarks is compensated by orbital angular momentum - here it is orbital angular momentum of the quarks and anti-quarks (the latter predominantly $\bar{u}$ in the p-wave).

\subsection{Summary of the Theoretical Corrections}

We have examined three corrections to the fraction of the spin of a nucleon carried by its quark and anti-quarks that arise naturally in any realistic treatment of proton structure:

- The valence quarks must be treated relativistically. For example, in the MIT bag model they satisfy the Dirac equation and the lower component of the Dirac spinor for a spin-up valence quark in an s-state will have predominantly $L_{z}=+1$ and spin down. This reduces the fraction of the spin carried by valence quarks from $100 \%$ to around $65 \%$.

- The Clebsch-Gordon algebra for the dominant Fock component of the nucleon wave function associated with its pion cloud is identical. That is, the dominant term has the quark core (or "bare nucleon", with spin down) while the pion has predominantly $L_{z}=+1$. Within the context of chiral quarks models, such as the CBM, this means that whatever the spin content within the quark model used, dressing it with the pion cloud reduces that value by a factor of order $(0.7,0.8)$.

- The exchange current correction associated with OGE in the proton also reduces the amount of spin carried by the quarks by about $15 \%$, where this number has so far only been calculated within the MIT bag model.

Clearly, if we combine these three corrections, that is we assume a relativistic description of the structure of the nucleon which incorporates OGE and chiral symmetry, the simple accounting of the 
fraction of the nucleon spin carried by its quarks and anti-quarks is:

$$
\begin{aligned}
\Sigma & =(0.7,0.8) \times(0.65-0.15) \\
& \rightarrow \Sigma \in(0.35,0.40)
\end{aligned}
$$

This result is in very satisfactory agreement with the current experimental values given earlier in Eq. (4) and the problem appears to be solved - at least at the level of a few percent.

There were two reasons why this conclusion could not be drawn in 1988. First the experimental target was $14 \%$ and possibly zero and even combined these corrections could not explain that. Secondly, at the time, the various studies of chiral quark models suggested that a large fraction of the mass splitting between the $\mathrm{N}$ and $\Delta$ arose from pion cloud corrections. If that were so, it would be double counting to include both the pion cloud correction and the OGE correction with a strength determined by the observed N- $\Delta$ mass splitting.

Experimental progress over the past 20 years has eliminated the first problem. For the second we owe a resolution to the sophisticated studies of hadron properties as a function of quark mass that have been stimulated by lattice QCD over the past decade. We review this briefly in the next section, showing that as a result of discoveries by Leinweber, Young and Thomas concerning the link between quenched and full lattice QCD, we now know that only $40 \pm 20 \mathrm{MeV}$ of the observed $300 \mathrm{MeV} \mathrm{N}-\Delta$ mass splitting comes from the pion cloud [41]. Consequently, there is little or no double counting as 80-90\% of the N- $\Delta$ mass difference would then come from OGE and the effective strong coupling constant, $\alpha_{s}$, used to calculate the OGE correction to the quark spin is appropriate.

\section{The Origin of the $\mathrm{N}-\Delta$ Mass Difference and Lattice QCD}

One of the unexpected but very positive consequences of our lack of supercomputing power is the fact that it has not been possible to compute physical hadron properties in lattice QCD. In fact, with computation time scaling like $m_{\pi}^{-9}$ (if we include the larger lattice size needed), calculations have covered the pion mass range from 0.3 to $1.0 \mathrm{GeV}$ (or higher). Far from being a disappointment, this has given us a wealth of unexpected insight into how QCD behaves as the light quark masses are varied [42]. In terms of the insight this has given us into hadron structure it is both truly invaluable and thus far under-utilized.

The most striking feature of the lattice data is that in the region $m_{\pi}>0.4 \mathrm{GeV}$, in fact for almost all of the simulations made so far, all baryon properties show a smooth dependence on quark mass, totally consistent with that expected within a constituent quark model. The rapid, non-linear dependence on $m_{\pi}$ required by the LNA and NLNA behavior of $\chi \mathrm{PT}$ are notably absent from the data!

The conventional view of $\chi \mathrm{PT}$ has no explanation for this simple, universal observation. Worse, in seeking to apply $\chi \mathrm{PT}$ to extrapolate the data back to the physical pion mass, it has been necessary to rely on ad hoc cancellations between the high order terms in the usual power series expansion (supplemented by the required non-analytic behavior). In fact, there is strong evidence that such series expansions have been applied well beyond their region of convergence [43] and that as a result such extrapolations are largely unreliable.

On the other hand, the picture of the pion cloud that we have discussed briefly yields a very natural explanation of the universal, constituent quark model behaviour of hadron properties found in the lattice simulations for $m_{\pi}>0.4 \mathrm{GeV}$. The natural high momentum cut-off on the momentum of the emitted pion, which is associated with the finite size (typically $R \sim 1 \mathrm{fm}$ ) of the bare baryon (i.e., the bag in the CBM), strongly suppresses pion loop contributions as $m_{\pi}$ increases. The natural mass scale which sets the boundary between rapid chiral variation and constituent quark type behavior is $1 / R \sim 0.2$ to 0.4 $\mathrm{GeV}$. Indeed, when in the early investigation of the quark mass dependence of nucleon properties the

CBM was compared directly with lattice data, the agreement was remarkably good [45]. (Similar results 

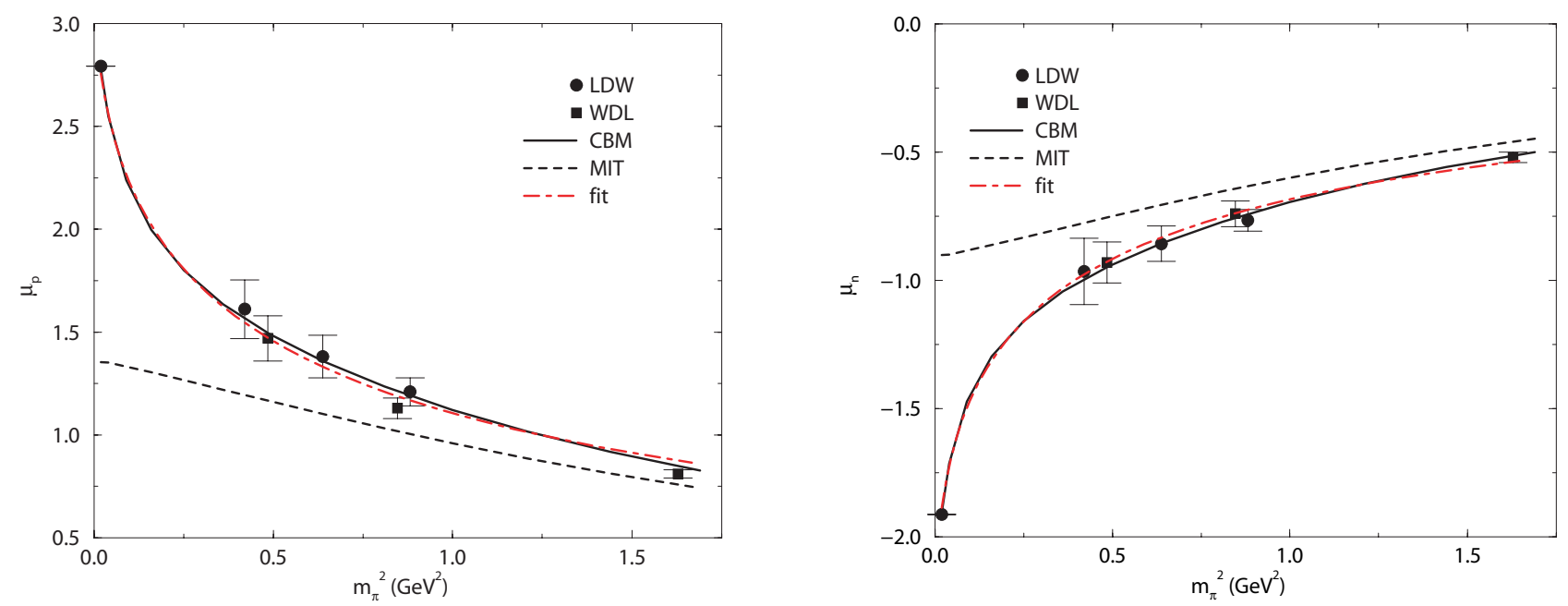

Figure 2: Comparison between early lattice QCD data for $\mu_{p}$ and $\mu_{n}$ and the CBM versus $m_{\pi}^{2}$ [44]. The model is also compared with a simple chiral fit and the MIT bag model (without the pion cloud).

have been obtained recently within the chiral quark soliton model [46.) We illustrate this in Fig. 2 for the magnetic moments of the proton and neutron. The results were equally as impressive for the $\mathrm{N}$ and $\Delta$ masses and magnetic moments, the proton charge radius and the moments of its parton distribution functions [47]. The key features necessary to reproduce the behaviour found at large quark mass in lattice QCD and to reproduce the experimentally measured data at the physical mass seem to be that:

- The treatment of the pion cloud (chiral) corrections ensures the correct LNA ( and NLNA, although in practice this seems less important in many applications) behaviour of QCD

- The pion cloud contribution is suppressed for $m_{\pi}$ beyond $0.4 \mathrm{GeV}$, and

- the underlying quark model exhibits constituent quark like behaviour for the corresponding range of current quark masses.

While the CBM satisfies all of these properties, in analyzing lattice QCD data one does not want to rely on any particular quark model. However, one does need to suppress the pion cloud as $m_{\pi}$ goes up, and the simple use of a finite range regulator (FRR) in the evaluation of the pion loops that yield the LNA and NLNA behaviour ensures this at the cost of one additional parameter, the cutoff mass $\Lambda$. If the data are good enough one can use this as a fitting parameter but in general it is sufficient [48] to choose a value consistent with the physical arguments presented above. The sensitivity of the extrapolation to the choice of the functional form of the FRR is then an additional source of systematic error in the final quoted result. In the case of the nucleon mass the corresponding systematic error was [49] of the order of a mere $0.1 \%$.

One of the most remarkable results of this physical understanding of the role of the pion cloud and, in particular, its suppression at large pion mass has been the unexpected discovery of a connection between lattice simulations based upon quenched QCD (QQCD) and full QCD [50]. In a study of the quark mass dependence of the $\mathrm{N}$ and $\Delta$ masses [41], it was discovered that if the self-energies appropriate to either QQCD or full QCD were regulated using the same dipole form for the FRR (the dipole being the most natural physical choice given that the axial form factor of the nucleon has a dipole form) with mass parameter $\Lambda=0.8 \mathrm{GeV}$ (the preferred value, as noted above), then the residual expansions for the nucleon mass in QQCD and QCD (and also for the $\Delta$ in QQCD and QCD) were the same within the errors of the fit! This is a remarkable result which a posteriori gives enormous support to the physical picture of the baryons consisting of confined valence quarks surrounded by a perturbative pion cloud. The baryon core is basically determined by the confinement mechanism and provided the choice of 
lattice scale reproduces the physically known confining force (either through the string tension or the Sommer parameter [51], derived from the heavy quark potential) it makes little difference whether one uses QQCD or full QCD to describe that core. What does matter is the change in the chiral coefficients as one goes from QQCD to full QCD.

Perhaps the most significant application of this discovery has been the application to the calculation of the octet magnetic moments and charge radii based on accurate QQCD simulations that extend to rather low quark mass. Using the constraints of charge symmetry this has led to some extremely accurate calculations of the strange quark contributions to the magnetic moment [52] and charge radius [53] of the proton. Indeed, those calculations are in excellent agreement with the current world data [54, 55] but, in a unique example in modern strong interaction physics, they are an order of magnitude more accurate.

In the present context, the key result of the analysis of QQCD and full QCD data for the N and $\Delta$ masses is that the contribution of the pion cloud to their mass difference is really under control. The result is that only $40 \pm 20 \mathrm{MeV}$ of the observed mass difference can be attributed to pions. The rest must be associated with other mechanisms such as the traditional OGE hypefine interaction.

\section{Summary and Discussion}

Two decades of experimental investigation have given us a wealth of important new information concerning the spin structure of the proton. We now know that the spin crisis is nowhere near as severe as once thought but still only about a third of the proton spin is carried by its quarks. Polarized gluons, which in principle could contribute through the axial anomaly, in practice seem to play no significant role. It seems likely that less than $5 \%$ of the missing spin can come from polarized glue and the sign may be such that it makes the problem slightly worse.

Instead it appears that important aspects of the non-perturbative structure of the nucleon $d o$ resolve the crisis. Indeed, three pieces of physics present in any realistic model are required. These consist of the relativistic motion of the valence quarks, the one-gluon-exchange interaction needed to describe the hyperfine splitting of hadron masses (especially the N- $\Delta$ mass difference) and finally the inclusion of the pion cloud required by chiral symmetry. These three terms reduce the fraction of the spin of the proton carried by its quarks to between 35 and 40\%, in excellent agreement with the latest experimental data. The theoretical consistency of this picture owes a great deal to recent studies of the dependence on quark mass of hadron properties calculated in lattice QCD.

For the future, it will be critical to test that the missing components of the proton spin do indeed reside as orbital angular momentum on the quarks and anti-quarks, as implied by this theoretical explanation. In this respect the program of measurements of Generalized Parton Distributions, especially following the $12 \mathrm{GeV}$ Upgrade at Jefferson Lab, will be vital [56, 57, 58].

\section{Acknowledgements}

I would like to thank both Andreas Schreiber and Fred Myhrer who were partners in the original work on the role of non-perturbative structure in the spin crisis. The collaboration with Steven Bass has been important to my understanding of the role of gluons in the spin problem. Finally, the insight into the behaviour of hadron properties as a function of quark mass owes a great deal to Derek Leinweber and Ross Young. This work was supported in part by U. S. DOE Contract No. DE-AC05-06OR23177, under which Jefferson Science Associates, LLC, operates Jefferson Lab. 


\section{References}

[1] A. W. Thomas and W. Weise, "The Structure of the Nucleon," Berlin, Germany: Wiley-VCH (2001) $389 p$

[2] S. D. Bass, "The Spin structure of the proton," World Scientific, 2007. (ISBN 978-981-270-946-2 and ISBN 978-981-270-947-9). $212 p$.

[3] J. Ashman et al. [European Muon Collaboration], Phys. Lett. B 206 (1988) 364.

[4] A. W. Schreiber and A. W. Thomas, Phys. Lett. B 215 (1988) 141.

[5] F. Myhrer and A. W. Thomas, Phys. Rev. D 38 (1988) 1633.

[6] S. L. Adler, Phys. Rev. 177 (1969) 2426.

[7] R. J. Crewther, Acta Phys. Austriaca Suppl. 19 (1978) 47.

[8] A. V. Efremov and O. V. Teryaev, Dubna preprint E2-88-287 (May 1988).

[9] G. Altarelli and G. G. Ross, Phys. Lett. B 212 (1988) 391.

[10] R. D. Carlitz, J. C. Collins and A. H. Mueller, Phys. Lett. B 214 (1988) 229.

[11] A. Chodos, R. L. Jaffe, K. Johnson and C. B. Thorn, Phys. Rev. D 10 (1974) 2599.

[12] I. C. Cloet, W. Bentz and A. W. Thomas, arXiv:0708.3246 [hep-ph].

[13] S. D. Bass et al., Phys. Rev. D 66 (2002) 031901 arXiv:hep-ph/0207071.

[14] S. D. Bass, Rev. Mod. Phys. 77 (2005) 1257 arXiv:hep-ph/0411005.

[15] S. D. Bass and A. W. Thomas, J. Phys. G 19 (1993) 925.

[16] S. D. Bass, B. L. Ioffe, N. N. Nikolaev and A. W. Thomas, J. Moscow. Phys. Soc. 1 (1991) 317.

[17] E. S. Ageev et al. [Compass Collaboration], Phys. Lett. B 647 (2007) 330 arXiv:hep-ex/0701014.

[18] E. S. Ageev et al. [COMPASS Collaboration], Phys. Lett. B 612 (2005) 154 arXiv:hep-ex/0501073].

[19] E. Leader, A. V. Sidorov and D. B. Stamenov, Phys. Rev. D 75 (2007) 074027 arXiv:hep-ph/0612360.

[20] K. V. Dharmawardane et al. [CLAS Collaboration], Phys. Lett. B 641 (2006) 11 arXiv:nucl-ex/0605028.

[21] P. Liebing [HERMES Collaboration], AIP Conf. Proc. 915 (2007) 331.

[22] E. S. Ageev et al. [COMPASS Collaboration], Phys. Lett. B 633 (2006) 25 arXiv:hep-ex/0511028].

[23] E. M. Kabuss [COMPASS Collaboration], AIP Conf. Proc. 870 (2006) 646.

[24] A. Bazilevsky [for the PHENIX Collaboration], invited talk at Pacific-SPIN 2007 (Vancouver).

[25] A. Adare et al. [PHENIX Collaboration], Phys. Rev. D 76 (2007) 051106 [arXiv:0704.3599 [hep-ex]].

[26] M. Sarsour [For the STAR Collaboration], contributed talk at the DNP Fall meeting (Newport News, Oct 2007).

[27] B. I. Abelev et al. [STAR Collaboration], arXiv:0710.2048 [hep-ex].

[28] A. Airapetian et al. [HERMES Collaboration], Phys. Rev. D 75 (2007) 012007 [Erratum-ibid. D 76 (2007) 039901] arXiv:hep-ex/0609039.

[29] V. Y. Alexakhin et al. [COMPASS Collaboration], Phys. Lett. B 647 (2007) 8 arXiv:hep-ex/0609038.

[30] F. Myhrer and A. W. Thomas, arXiv:0709.4067 [hep-ph].

[31] D. B. Leinweber, A. W. Thomas and R. D. Young, Phys. Rev. Lett. 86 (2001) 5011 arXiv:hep-ph/0101211.

[32] A. W. Thomas, Prog. Theor. Phys. Suppl. 168 (2007) 614; arXiv:0711.2259 [nucl-th].

[33] S. Theberge et al., Phys. Rev. D 22, 2838 (1980) [Erratum-ibid. D 23, 2106 (1981)]. 
[34] A. W. Thomas, Adv. Nucl. Phys. 13, 1 (1984).

[35] J. Speth and A. W. Thomas, Adv. Nucl. Phys. 24, 83 (1997).

[36] W. Melnitchouk, J. Speth and A. W. Thomas, Phys. Rev. D 59 (1999) 014033 arXiv:hep-ph/9806255.

[37] A. W. Thomas, Phys. Lett. B 126, 97 (1983).

[38] M. Arneodo et al. [New Muon Collaboration], Nucl. Phys. B 487 (1997) 3 arXiv:hep-ex/9611022].

[39] A. De Rujula, H. Georgi and S. L. Glashow, Phys. Rev. D 12 (1975) 147.

[40] H. Hogaasen and F. Myhrer, Phys. Lett. B 214 (1988) 123.

[41] R. D. Young et al., Phys. Rev. D 66 (2002) 094507 arXiv:hep-lat/0205017.

[42] A. W. Thomas, Nucl. Phys. Proc. Suppl. 119, 50 (2003) arXiv:hep-lat/0208023.

[43] D. B. Leinweber, A. W. Thomas and R. D. Young, Nucl. Phys. A 755, 59 (2005)

[44] D. B. Leinweber, D. H. Lu and A. W. Thomas, Phys. Rev. D 60, 034014 (1999)

[45] D. B. Leinweber et al., Phys. Rev. D 61, 074502 (2000) arXiv:hep-lat/9906027.

[46] K. Goeke et al., Eur. Phys. J. A 27, 77 (2006); K. Goeke et al., arXiv:hep-ph/0702031.

[47] W. Detmold, W. Melnitchouk and A. W. Thomas, Phys. Rev. D 66, 054501 (2002)

[48] G. P. Lepage, arXiv:nucl-th/9706029.

[49] D. B. Leinweber, A. W. Thomas and R. D. Young, Phys. Rev. Lett. 92, 242002 (2004)

[50] S. Aoki et al. [CP-PACS Collaboration], Phys. Rev. Lett. 84, 238 (2000)

[51] R. Sommer, Nucl. Phys. B 411, 839 (1994) [arXiv:hep-lat/9310022].

[52] D. B. Leinweber et al., Phys. Rev. Lett. 94, 212001 (2005)

[53] D. B. Leinweber et al., Phys. Rev. Lett. 97, 022001 (2006)

[54] R. D. Young et al., Phys. Rev. Lett. 97, 102002 (2006) arXiv:nucl-ex/0604010.

[55] R. D. Young, R. D. Carlini, A. W. Thomas and J. Roche, Phys. Rev. Lett. 99 (2007) 122003 [arXiv:0704.2618 [hep-ph]].

[56] The Science and Experimental Equipment for the $12 \mathrm{GeV}$ Upgrade of CEBAF http://www.jlab.org/12GeV/CDR_for_NSAC_Town_Meeting_r3.pdf

[57] M. Guidal, M. V. Polyakov, A. V. Radyushkin and M. Vanderhaeghen, Phys. Rev. D 72 (2005) 054013 arXiv:hep-ph/0410251].

[58] A. V. Belitsky and A. V. Radyushkin, Phys. Rept. 418 (2005) 1 arXiv:hep-ph/0504030]. 